\title{
Determinación de la longitud de transferencia de alambres con distintos grados de corrosión en elementos pretensados de hormigón
}

\author{
Determination of wires transfer length in prestressed concrete members \\ with different levels of corrosion
}

$\underline{\text { P. Anaya }}^{(*)}$, J. Rodríguez ${ }^{(* *)}$, C. Andrade ${ }^{(* * *)}$, B. Martín-Pérez ${ }^{(* * * *)}$, C. López Hombrados ${ }^{(* * * * *)}$

\section{RESUMEN}

En elementos pretensados de hormigón, la adherencia entre el acero y el hormigón puede verse deteriorada por diferentes motivos, siendo uno de los más recurrentes el daño inducido por procesos de corrosión. El parámetro fundamental para caracterizar las propiedades adherentes existentes entre el acero pretensado y el hormigón es la longitud de transferencia. Este artículo compara los resultados experimentales obtenidos en ensayos sobre elementos pretensados tipo viga con las predicciones arrojadas por un modelo analítico, basado en la teoría de cilindros de pared gruesa y en la fisuración cohesiva del hormigón, desarrollado para analizar los procesos de degradación de la adherencia motivados por la corrosión en alambres lisos e indentados y su influencia en el comportamiento de elementos pretensados de hormigón.

Palabras clave: Longitud de transferencia, longitud de transmisión, hormigón pretensado, adherencia, corrosión, modelo analítico.

\section{ABSTRACT}

Bond between steel and concrete in prestressed concrete members may be degraded due to different reasons, being one of the most frequent ones the corrosion induced damage. Transfer length is the key indicator to describe the bond properties between prestressed steel and concrete. Through tests on prestressed beams, this paper is focused on the experimental verification of an analytical model developed to analyse the processes of bond degradation due to the corrosion of both indented and smooth wires, and its influence on the behaviour of prestressed concrete members. The model is based on both the thick-walled cylinder theory and the cohesive cracking for concrete.

Keywords: Transfer length, transmission length, prestressed concrete, bond, corrosion, analytical model.

(*) Dr. Arquitecto. Universidad Politécnica de Madrid (UPM), Madrid (España).

(**) Dr. Ingeniero de Caminos. Profesor Titular. Universidad Politécnica de Madrid (ETSAM-UPM), Madrid (España).

(***) Dra. Química Industrial. Investigadora. Centro Intern. de Métodos Numéricos en Ingeniería (CIMNE), Madrid (España).

$(* * *)$ Ph.D. Civil Engineering. Associate Professor. University of Ottawa, Ottawa (Canada).

$(* * * *)$ Ingeniero de Caminos. Vicedirector. Instituto de ciencias de la construcción Eduardo Torroja (IETcc-CSIC).

Persona de contacto/Corresponding author: pabloanayagil@gmail.com (P. Anaya).

ORCID: https://orcid.org/oooo-0oo2-0953-4952 (P. Anaya); https://orcid.org/oooo-0oo2-7272-3772 (J. Rodríguez); https://orcid.org/oooo-0003-2374-0928 (C. Andrade); https://orcid.org/oooo-0oo2-9655-511X (B. Martín-Pérez); https://orcid.org/oooo-0001-6084-6428 (C. López Hombrados).

Cómo citar este artículo/Citation: Anaya, P.; Rodríguez, J.; Andrade, C.; Martín-Pérez, B.; López Hombrados, C. (2020). Determinación de la longitud de transferencia de alambres con distintos grados de corrosión en elementos pretensados de hormigón. Informes de la Construcción, 72(558): e339. https://doi.org/10.3989/ic.71428.

Copyright: (C) 2020 CSIC. Este es un artículo de acceso abierto distribuido bajo los términos de la licencia de uso y distribución Creative Commons Reconocimiento 4.0 Internacional (CC BY 4.0). 


\section{INTRODUCCIÓN}

En elementos pretensados de hormigón, la adherencia entre el acero y el hormigón es una propiedad esencial para garantizar la integridad de la estructura. El pretensado tracciona el acero y comprime el hormigón, permitiendo una mejora del comportamiento del elemento tanto en servicio (ELS de deformación y ELS de fisuración) como en rotura (ELU de agotamiento frente a esfuerzo cortante y frente a momento flector). Para ello, es crucial la existencia de tensiones de adherencia entre los alambres y el hormigón, asegurando la transmisión de las fuerzas de pretensado tras el destesado, y el anclaje de éstos durante la vida útil del elemento. Esta adherencia puede verse deteriorada por diferentes motivos, siendo uno de los más recurrentes el daño inducido por corrosión. Los productos de corrosión, cuyo volumen específico es mayor que el del acero original, generan tensiones en el hormigón que circunda el alambre y propician la aparición de fisuras y desprendimientos en el recubrimiento, reduciendo su capacidad de confinamiento y, consecuentemente, mermando las propiedades adherentes entre el hormigón y el acero $(1,2)$.

El parámetro fundamental para caracterizar las propiedades adherentes existentes entre el acero pretensado y el hormigón es la longitud de transferencia, definida como aquella necesaria para que la totalidad de la fuerza de pretensado introducida en una armadura pretesa se transfiera por adherencia al hormigón. Las expresiones recogidas en la mayoría de los códigos estructurales para la caracterización de dicha longitud suelen considerar únicamente el efecto de algunas variables $(3,4,5,6)$, como el diámetro del alambre o la tensión de pretensado inicial, omitiendo otros parámetros, como la resistencia del hormigón o el espesor de recubrimiento, cuya influencia ha sido demostrada en numerosos trabajos $(7,8,9)$. Además, en ningún caso, se cuantifica cómo afecta el ataque por corrosión a dicha longitud. Por tanto, es patente la necesidad de desarrollar un método racional que permita evaluar la adherencia y, consecuentemente, la longitud de transferencia, en elementos pretensados de hormigón con distintas configuraciones geométricas y mecánicas, así como con distintos grados de corrosión.

Este artículo detalla una parte del trabajo de investigación desarrollado en colaboración entre el Instituto de ciencias de la construcción Eduardo Torroja y la Universidad de Ottawa, recogido en una tesis doctoral elaborada por el primer autor de este artículo (10), en el que se han analizado los procesos de degradación de la adherencia motivados por la corrosión en alambres lisos e indentados y su influencia en el comportamiento de elementos pretensados de hormigón. La metodología teórico-experimental elegida se ha mostrado como un procedimiento eficaz para identificar, analizar y cuantificar las principales variables mecánicas y geométricas que intervienen en el fenómeno de la corrosión en elementos pretensados. Las etapas de las que ha constado esta investigación son las siguientes:

1) Desarrollo de un modelo analítico bidimensional para la determinación de las tensiones radiales a nivel de sección derivadas de los procesos de corrosión en estructuras de hormigón.

2) Realización de campaña de ensayos de arrancamiento/pull-out (11) para la calibración de los parámetros que definen la expansión de los productos de corrosión y las propiedades adherentes entre el hormigón y el acero.

3) Extensión del modelo a la dirección longitudinal del elemento armado (tres dimensiones) para la determinación de las leyes que rigen la relación entre adherencia y deslizamiento, en alambres sanos y corroídos, mediante su comparación con la campaña experimental anterior.

4) Elaboración de un modelo analítico integral para la determinación de la longitud de transferencia en estructuras pretensadas sanas y corroídas, acoplando los efectos del pretensado al modelo analítico tridimensional sobre elementos de hormigón con corrosión.

5) Verificación del modelo integral mediante su comparación con ensayos sobre elementos pretensados con procesos de degradación por corrosión (12).

6) Desarrollo de expresiones simplificadas, basadas en el modelo analítico verificado, para el cálculo de longitudes de transferencia en elementos pretensados de hormigón tanto con alambres sanos como corroídos, comparando los resultados obtenidos con campañas experimentales de otros investigadores y con los códigos estructurales cuyo uso está más extendido.

El presente artículo se centra en la etapa 5), la verificación del modelo analítico mediante su comparación con los resultados obtenidos en una campaña experimental sobre elementos pretensados tipo viga diseñada a tal efecto.

\section{DESCRIPCIÓN DEL MODELO ANALÍTICO}

En el modelo propuesto, el mecanismo adherente se desacopla en dos componentes diferenciadas (Figura 1):

- Una componente transversal $\sigma_{r}$ (o radial) que puede ser descompuesta en el sumatorio de las siguientes tensiones:

- Tensión derivada del estiramiento del alambre (efecto Hoyer) y de las tracciones perpendiculares a las compresiones introducidas por el pretensado en el hormigón, $\sigma_{r, p}$.

- Tensión radial por la acumulación de productos de corrosión, $\sigma_{r, \text { corr }}$.

- Tensión provocada por fuerzas de confinamiento exteriores, $\sigma_{r, \text { conf }}$.

- Tensión radial provocada por la retracción, $\sigma_{r, s h}$.

- Una componente longitudinal $\tau_{b}$, que reproduce la variación de las tensiones a lo largo del alambre, transferidas gradualmente al hormigón circundante cuando la fuerza de pretensado es liberada tras el endurecimiento del hormigón. Esta componente es la que tiene en cuenta los fenómenos que tienen lugar en la dirección longitudinal del alambre, tales como la pérdida de adherencia y el deslizamiento relativo del alambre.

Estas dos componentes del modelo, $\tau_{b}$ y $\sigma_{r}$, dejando a un lado la adhesión química $\tau_{a d h}$, que suele considerarse cons- 


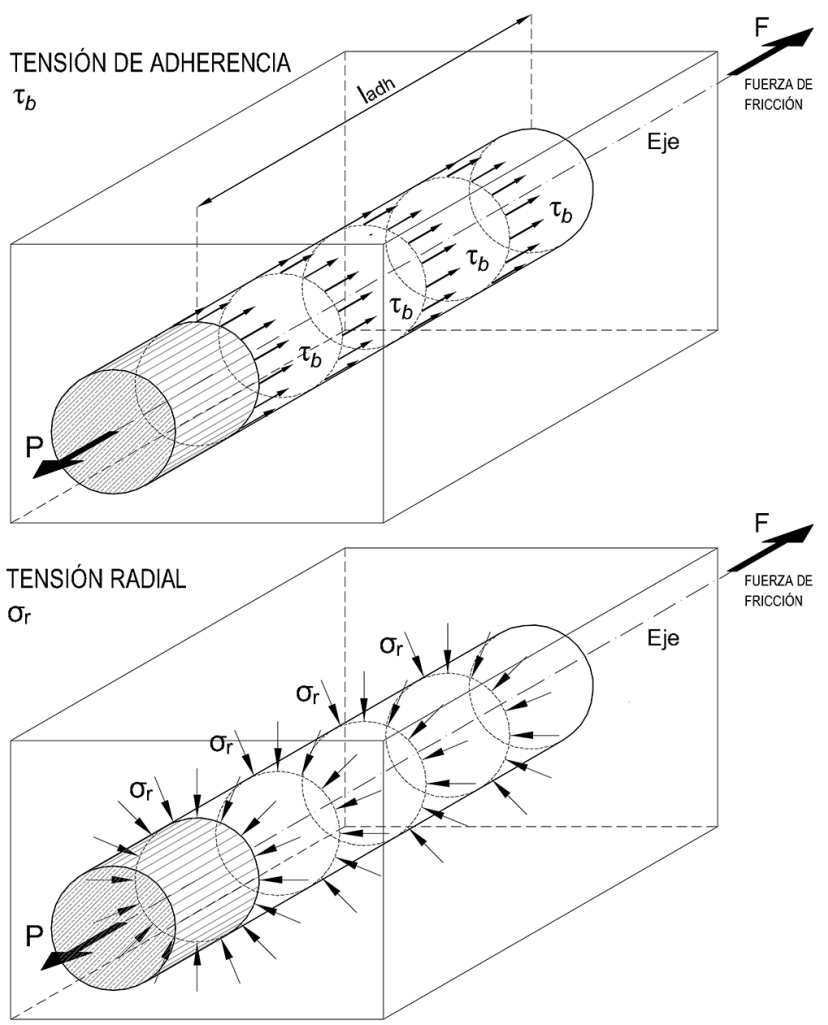

Figura 1. Tensiones tangenciales $\tau_{\mathrm{b}}$ (arriba) y radiales $\sigma_{\mathrm{r}}$ (abajo) a lo largo de la superficie cilíndrica del alambre.

tante y cuya acción desaparece para deslizamientos muy pequeños, se encuentran vinculadas a través del coeficiente de fricción $\mu[1]$ :

$$
\tau_{b}=\mu \sigma_{r}
$$

Sustituyendo la tensión radial total $\sigma_{r}$ por el conjunto de tensiones que la conforman, y añadiendo la tensión de adherencia debida al mecanismo de adhesión $\tau_{a d h}$, se obtiene la expresión que gobierna el comportamiento adherente de alambres pretensados embebidos en hormigón [2]:

$$
\tau_{b}=\mu\left[\sigma_{r, p}+\sigma_{r, c o r r}+\sigma_{r, s h}+\sigma_{r, c o n f}\right]+\tau_{a d h}
$$

El modelo desarrollado se compone de tres fases diferenciadas que se detallan en los siguientes subapartados: determi- nación de las tensiones radiales (Fase I), simulación de los ensayos de arrancamiento o pull-out (Fase II) y determinación de la longitud de transferencia (Fase III).

\subsection{Fase I: Determinación de las tensiones radiales}

En el modelo, el estado tensional provocado por la presión interna derivada de la corrosión y del pretensado ha sido establecido gracias a la solución del cilindro de pared gruesa bajo presión interna uniforme, basada en la teoría de la elasticidad lineal para deformaciones planas (13). Las primeras aplicaciones de esta solución a modelos para la determinación de tensiones en el hormigón fueron llevadas a cabo por Tepfers (14) y Bazant (15), tratando de predecir la fisuración del hormigón en secciones con tensiones radiales conocidas.

Una limitación importante de los modelos basados en los cilindros de pared gruesa es su incapacidad para tener en cuenta el comportamiento no lineal del hormigón, que toma relevancia con el comienzo de la fisuración radial a nivel de la interfaz acero-hormigón, cuando la presión interna alcanza un determinado valor. En este trabajo, para sortear esta limitación, la caracterización de la región fisurada se basa en un modelo de hormigón como material anisótropo, con fisuración cohesiva $(16,17,18)$. Por tanto, la fisuración se modela como un proceso de ablandamiento del hormigón que comienza cuando a nivel de la interfase las tensiones circunferenciales $\sigma_{\theta}$ superan la resistencia a tracción del hormigón $f_{\mathrm{ct}}$, y finaliza cuando la deformación circunferencial alcanza un determinado valor último, con tensiones residuales nulas. Este planteamiento permite establecer tres estados diferenciados del cilindro de pared gruesa en el proceso de fisuración: -cilindro sin fisurar, cilindro parcialmente fisurado y cilindro totalmente fisurado (Figura 2).

Esta fase del modelo ha permitido determinar, por un lado, las tensiones radiales $\sigma_{r}$ a lo largo del recubrimiento, para unas condiciones de nivel de pretensado y corrosión, y por otro, predecir la apertura de fisuras y el avance de éstas. El análisis depende tanto de parámetros conocidos, tales como las propiedades geométricas y mecánicas de los materiales, como de parámetros cuyo valor debe ser calibrado mediante la comparación de los resultados teóricos con los obtenidos en la campaña de ensayos tipo pull-out. Esta campaña, que ha constado de 109 ensayos de arrancamiento, en la que se han modificado variables como el acabado superficial de los alambres, la relación recubrimiento/diámetro o el grado de (a) Recubrimiento sin fisurar

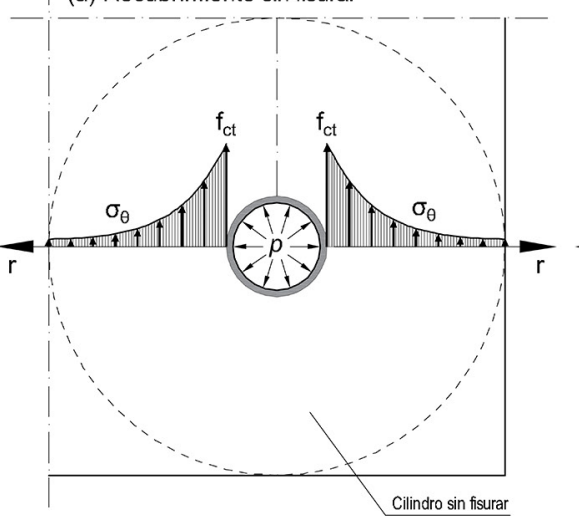

(b) Recubrimiento parcialmente fisurado

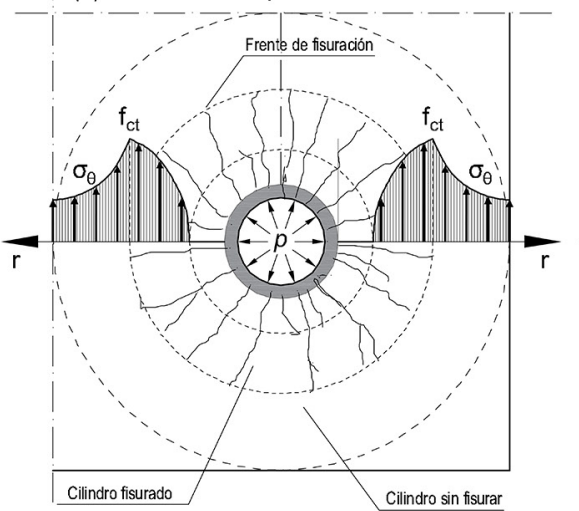

(c) Recubrimiento totalmente fisurado

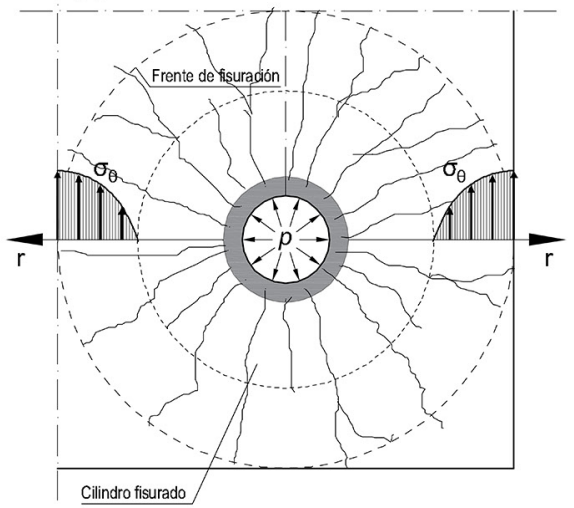

Figura 2. Diferentes estados de fisuración del recubrimiento: a) hormigón sin fisurar; b) hormigón parcialmente fisurado y c) hormigón totalmente fisurado. 
pérdida de sección por corrosión, ha permitido calibrar los siguientes parámetros:

- Coeficiente de fricción $\mu$ : 0,65;

- Coeficiente de expansión de óxidos n: 2;

- Módulo de compresibilidad volumétrica $K r:$ o,5 GPa;

- Espesor de la zona porosa $t_{p}$ : nuevo modelo de distribución parabólica, inspirada en las leyes de difusión de Fick, en el que el porcentaje de óxidos que actúa sobre el recubrimiento se incrementa hasta alcanzar el máximo a una distancia $t_{p, c r i}$ de la interfaz acero-hormigón $\left(t_{p, c r i}=75 \mu \mathrm{m}\right)$;

- Volumen de óxidos en fisuras: El volumen de óxidos que penetra en las fisuras es igual para la misma densidad de corriente de corrosión $i_{\text {corr }}$ e independiente del espesor $c$ de recubrimiento.

\subsection{Fase II: Simulación del ensayo de arrancamiento}

En esta fase se ha llevado a cabo un análisis en tres dimensiones en el que, partiendo de las tensiones radiales $\sigma_{r}$ de la sección transversal, halladas en la etapa anterior, y reproduciendo las condiciones específicas del ensayo de pull-out, se analiza el comportamiento mecánico en la dirección longitudinal del elemento. Para ello, se recurre a los dos parámetros fundamentales que permiten el acople de ambas direcciones: el coeficiente de fricción $\mu$, calibrado en la Fase I, y la ley de adherencia "local"-deslizamiento $\tau_{b}-s$. Partiendo de una ley de adherencia-deslizamiento genérica se implementan las expresiones que permiten relacionar los deslizamientos $s$ que provoca la fuerza de arrancamiento, con la distribución de la tensión de adherencia $\tau_{b}$ a lo largo de la longitud adherente del alambre $l_{a d h}$. Posteriormente, mediante la comparación con las gráficas de adherencia "media"-deslizamiento $\tau_{b, \max }-s$ obtenidas de los ensayos de arrancamiento, se calibran las leyes genéricas de adherencia "local"-deslizamiento $\tau_{b}-s$, tanto de alambres lisos como indentados, obteniéndose el valor de los parámetros que las particularizan.

\subsection{Fase III: Determinación de la longitud de transferencia}

Una vez determinada la tensión radial en la sección transversal procedente de la transferencia del pretensado, de la ex- pansión de los productos de corrosión, de la retracción y de las fuerzas externas de confinamiento, y calibrados mediante ensayos el coeficiente de fricción y la ley de adherencia localdeslizamiento, es posible determinar la longitud de transferencia del pretensado $l_{t}$ para unas determinadas condiciones geométricas, mecánicas y/o de deterioro.

Las ecuaciones de equilibrio han sido establecidas y resueltas para cada sucesivo segmento en la dirección longitudinal de la viga. Las longitudes de transferencia se han determinado gracias a los perfiles de deformación de los elementos pretensados.

La longitud de transferencia $l_{t}$ depende de la distribución de las tensiones de adherencia $\tau_{b}$ a lo largo del alambre, y ésta a su vez depende de la tensión radial $\sigma_{r}$, del coeficiente de fricción $\mu$ y de la tensión debida al mecanismo de adhesión $\tau_{\text {adh }}$.

Tal y como se muestra en la Figura 3, la longitud del elemento pretensado $L$ puede ser discretizada en elementos de longitud $\Delta$ z. En cada uno de dichos elementos discretos, la tensión de adherencia $\tau_{b}$ es conocida, por lo que estableciéndose el equilibrio en su dirección longitudinal z puede deducirse el incremento de tensión normal $\Delta \sigma_{s z}$ que experimenta el alambre, de radio $r_{b}$ y sección $A_{s}$, en la transferencia del pretensado [3]:

$$
\Delta \sigma_{s z}=\pi 2 r_{b} \Delta z \tau_{b} / A_{s}
$$

Por tanto, dado que la tensión normal en el extremo del alambre de pretensado $(\mathrm{z}=\mathrm{O})$ es nula, el valor de dicha tensión en un elemento n-ésimo $\Delta \sigma_{\mathrm{s} z, n}$ será equivalente al sumatorio de incrementos de tensión hasta dicho elemento, tal y como sigue [4]:

$$
\sigma_{s z, n}=\sum_{o}^{n} \Delta \sigma_{s z}
$$

Al mismo tiempo, la variación de la deformación del alambre con respecto a la deformación provocada por el pretensado inicial será máxima en el extremo del elemento, $\mathrm{z}=\mathrm{O}$, por lo que para cualquier elemento discreto del elemento pretensada se cumple que [5]:

$[5]$ $\Delta \varepsilon_{s z, n}=\varepsilon_{s, 0}-\sigma_{s z, n} / E_{s}$

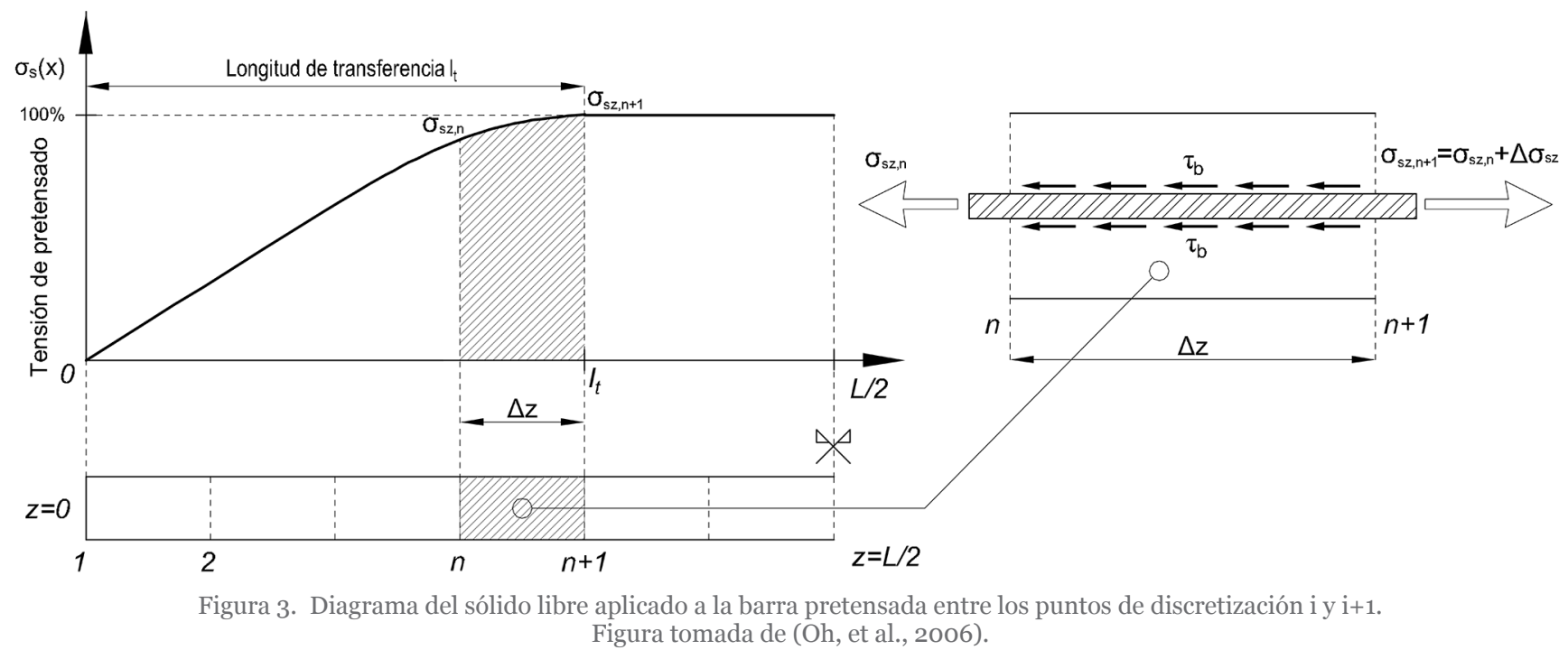


siendo $E_{\mathrm{s}}$ el módulo de elasticidad del acero. Conocida la tensión del alambre en un determinado punto, la deformación del hormigón $\varepsilon_{c z, n}$ en el mismo puede obtenerse de forma inmediata. Finalmente, la longitud de transferencia $l_{t}$ puede ser determinada como la distancia desde el extremo del alambre hasta el punto en el que la variación de la deformación en el alambre de pretensado $\Delta \varepsilon_{\mathrm{sz}, n}$, obtenido de la ecuación [5], se iguala con la deformación del hormigón $\varepsilon_{c z, n}$, es decir, el punto en el que se cumple que $\Delta \varepsilon_{\mathrm{sz}, n}=\varepsilon_{c z, n}$ (Figura 4). Con todo ello, queda definida la longitud de transferencia $l_{t}$, que será verificada mediante ensayos sobre vigas pretensadas reales en los próximos apartados.

\section{CAMPAÑA EXPERIMENTAL}

La campaña de ensayos que se presenta a continuación ha permitido la verificación experimental del modelo analítico propuesto en este trabajo. Dicha campaña ha constado de dos etapas diferenciadas: la primera de ellas se ha diseñado siguiendo la Norma UNE 7-436-82 (12) y su objetivo ha sido el de determinar las longitudes de transferencia en vigas pretensadas de hormigón con alambres sanos; la segunda, partiendo de las vigas anteriores, ha analizado la influencia de la corrosión en las propiedades adherentes.

La campaña ha constado de 4 vigas de hormigón, fabricadas mediante moldes metálicos, que han sido pretensadas mediante 4 alambres indentados de $5 \mathrm{~mm}$ de diámetro situados en cada una de las esquinas de la viga. Las vigas tenían una longitud de $2 \mathrm{~m}$, y una sección transversal de $80 x 90 \mathrm{~mm}^{2}$, con un recubrimiento mecánico de $20 \mathrm{~mm}$.

\subsection{Materiales}

El hormigón utilizado para la fabricación de las vigas contenía cemento CEMI 52,5R, una relación agua cemento a/c= 0,5 y áridos silíceos de $12 \mathrm{~mm}$ de tamaño máximo. Para la caracterización del hormigón se fabricaron probetas cúbicas de $150 \mathrm{~mm}$ de lado, obteniéndose una resistencia media a compresión de $28 \mathrm{MPa}$ a los 7 días y de $36 \mathrm{MPa}$ a los 35 días.

Se han utilizado alambres indentados de acero Y1860C, de $5 \mathrm{~mm}$ de diámetro, con una carga última de rotura de $f_{p, u}=1860 \mathrm{~N} / \mathrm{mm}^{2}$ y un módulo de elasticidad $E_{s}$ de $200000 \mathrm{~N} / \mathrm{mm}^{2}$. Los alambres se tesaron inicialmente hasta alcanzar valores cercanos a su límite elástico. Tras las pérdidas por penetración de cuñas, se recuperó carga mediante un sistema de tuercas, hasta lograr la fuerza deseada de $26,5 \mathrm{kN}$ por alambre, equivalente a una tensión de $1350 \mathrm{~N} / \mathrm{mm}^{2}$. Esta tensión supone hacer trabajar al alambre a un 72,5\% de la carga unitaria máxima $f p, u$, menor al $75 \%$ marcado por la EHE-o8 (4).

\subsection{Proceso de fabricación}

La bancada de pretensado, ubicada en la Nave de Ensayos Mecánicos del IETcc y de 12 metros de longitud, estaba limitada en cada extremo por estribos de anclaje, actuando uno como anclaje activo y el ubicado en el extremo opuesto como anclaje pasivo. Tras asegurar la posición de los alambres en el estribo pasivo de la bancada con cuñas de anclaje, éstos se tesaron mediante un gato hidráulico contra el estribo activo (Figura 5). Una vez comprobado el correcto trazado de los alambres respecto a la sección del prisma, se procedió al hormigonado de las vigas. Tras su fabricación, las vigas permanecieron 24 horas en los moldes, con un elevado porcentaje

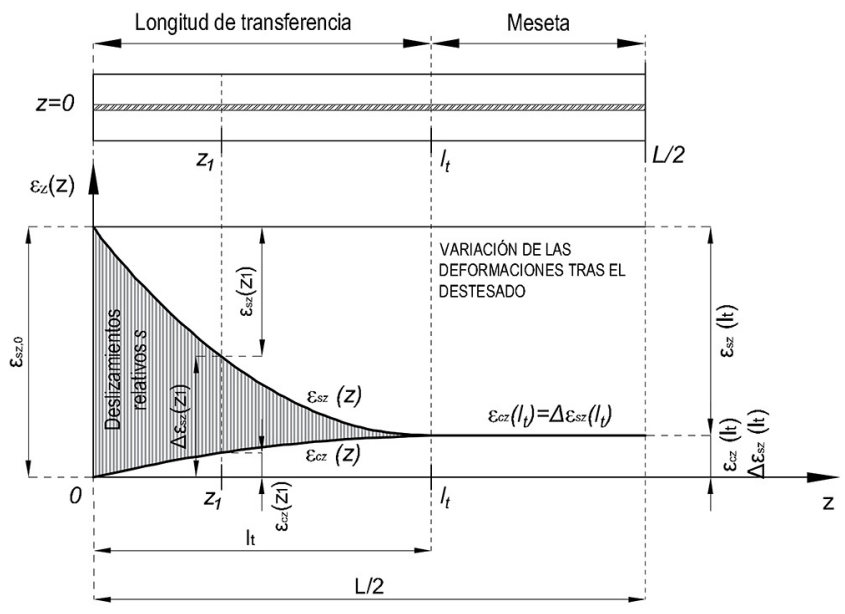

Figura 4. Variación de las deformaciones en el hormigón y en el acero a lo largo de la longitud de transferencia.
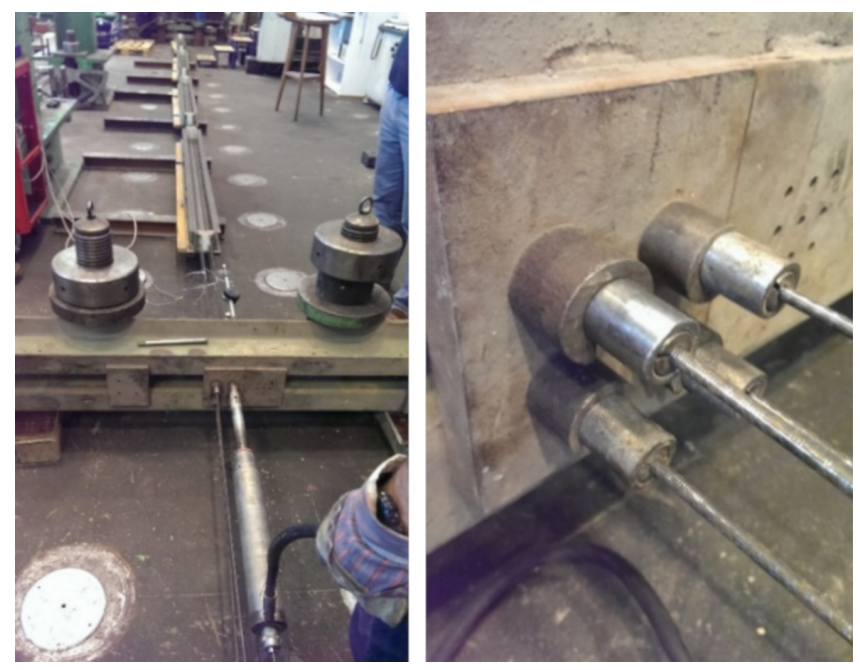

Figura 5. Bancada de pretensado y anclajes de los alambres.

de humedad. Una vez desmoldadas, se mantuvieron durante 7 días en condiciones de alta humedad (cubiertas con arpillera húmeda). Hasta el destesado de los alambres, a los 21 días tras el hormigonado, las vigas permanecieron en el banco de pretensado en ambiente de laboratorio. Tras la transferencia del pretensado, las vigas permanecieron almacenadas en condiciones de temperatura y de humedad ambiente, hasta la finalización de los ensayos.

\subsection{Instrumentación de las vigas pretensadas}

Tras el desmoldado y antes de la transferencia del pretensado, las vigas fueron instrumentadas. La determinación de la longitud de transferencia del pretensado se ha llevado a cabo mediante la monitorización de las deformaciones longitudinales en el hormigón $\varepsilon_{c}$ a lo largo de la viga. Para ello, se han utilizado dos sistemas complementarios (Figura 6): galgas extensométricas en una de las caras y puntos de medida para extensometría manual en la cara opuesta. A pesar de que estrictamente sólo sería necesario la determinación de las deformaciones longitudinales en uno de los paramentos verticales de la viga, se ubicaron en ambos laterales, con el fin de compensar posibles excentricidades de los esfuerzos de pretensado y minimizar así posibles errores. Para ello, sobre los ejes de cada una de las caras verticales de la viga, se ubicaron las galgas extensométricas y las bases de medida, a intervalos 


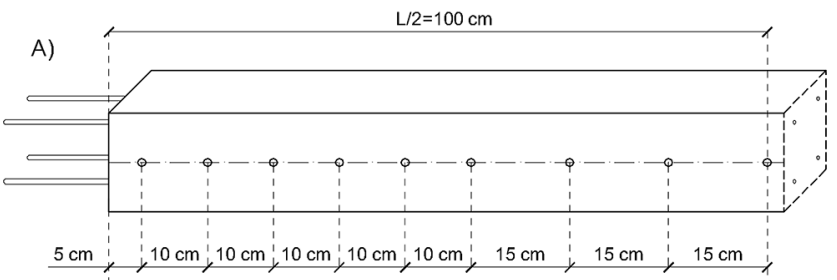

B)

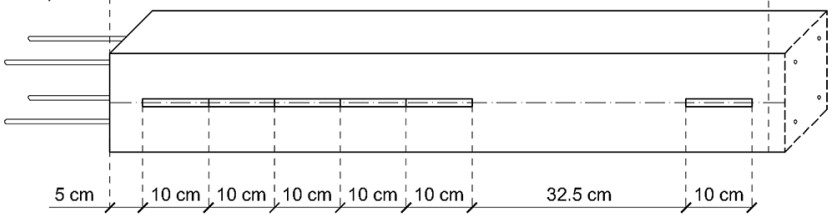

Figura 6. A) Ubicación de los puntos de medida para extensometría manual y B) Distribución de las galgas extensométricas.

regulares de longitud. Siguiendo la recomendación de la Norma UNE 7-436-82 (12), en los extremos de las vigas, los puntos de medida se dispusieron de forma que se pudieran obtener como mínimo cinco lecturas sobre la previsible longitud de transferencia $l_{t}$ del pretensado.

\subsection{Proceso de corrosión de los alambres}

Trascurrido un año desde la transferencia del pretensado, los alambres embebidos en las vigas fueron sometidos a un proceso de corrosión acelerada. El hormigón utilizado para la elaboración de las vigas no contaba con cloruros incorporados, por lo que, para facilitar el proceso de corrosión acelerada, se optó por forzar la penetración de cloruros desde la superficie del hormigón, basándose en el denominado "método integral” de la Norma UNE 83992-2:2012 EX (19, 20).

En términos generales, este ensayo consiste en exponer a la viga de hormigón, en la que se han embebido los alambres pretensados de acero, a un campo eléctrico aplicado mediante electrodos en sus lados opuestos, de manera que se fuerce la penetración de los cloruros presentes en una disolución en contacto con el hormigón, hasta alcanzar la posición de los alambres, posibilitando su despasivación (Figura 7). Según el Metodo Integral Acelerado, la despasivación tiene lugar cuando se detecta un potencial de -300 mV o más negativo. Una vez detectado dicho potencial, se comenzó el procedimiento de corrosión acelerada, para lo que se empleó un método galvanostático (1)(2). Este método consiste en aplicar una corriente constante, generada mediante un intensiostato, a través de un circuito en el que los alambres actúan como ánodos y sendas mallas de acero



Figura 7. Método Integral Acelerado sobre las vigas pretensadas. inoxidable situadas en la superficie superior e inferior de la viga funcionan como cátodo o contraelectrodo. Para asegurar la continuidad electrolítica se coloca una esponja permanentemente humedecida entre la superficie del hormigón y la malla de acero inoxidable.

Se ha aplicado una densidad de corriente de $100 \mu \mathrm{A} / \mathrm{cm}^{2}$, que se corresponde con la máxima densidad de corriente registrada en procesos de corrosión natural por cloruros (1)(2). Este valor de corriente permite conciliar unos tiempos de ensayo razonablemente cortos con valores no demasiado alejados de aquellos que pueden darse en la práctica.

\section{RESULTADOS}

Las longitudes de transferencia, tanto sobre las vigas con alambres sanos como sobre las vigas con procesos de deterioro por corrosión, han sido determinadas mediante la medición de las deformaciones en la superficie del hormigón. El método utilizado es el denominado "Método del 95\% de la deformación máxima media" (21), en el que la longitud de transferencia es definida como la mínima distancia desde el extremo hasta el punto en el que se alcanza el $95 \%$ de la deformación constante media en el centro del elemento.

Para todas las vigas, se han tomado medidas de las deformaciones de compresión en el hormigón durante 90 días, de manera continuada mediante las galgas extensométricas y a intervalos regulares de tiempo mediante los puntos de extensometría manual. Tal y como indica la Norma UNE 7-436-82 (12), dada la presumible heterogeneidad y el número de incertidumbres de los resultados obtenidos mediante este ensayo, éstos deben analizarse como el promedio de los obtenidos en al menos tres vigas, y no tanto de manera individualizada.

\subsection{Longitud de transferencia en alambres sanos}

En vigas pretensadas con alambres sanos, la deformación total del hormigón es el resultado del efecto acumulado de la deformación instantánea tras el pretensado ( $\mathrm{t}=\mathrm{O}$ días) y de las deformaciones debidas a los efectos dependientes del tiempo: la retracción del hormigón, que es constante a lo largo de toda la viga pretensada; la fluencia originada por la fuerza de compresión actuante en cada sección de la viga, causada por el pretensado, y la relajación del acero.

En la Figura 8 se presentan los diagramas de deformación longitudinal promedio de las cuatro vigas en los distintos periodos de tiempo considerados. Para su obtención se ha supuesto un comportamiento lineal de la ley de deformaciones a lo largo de la longitud de transferencia $l_{t}$, y un valor constante a lo largo de la meseta central, equivalente al $95 \%$ del valor medio de deformación $\varepsilon_{\max }$ obtenido en los ensayos. Tal y como se aprecia en la Figura 8, las deformaciones máximas en la meseta central $\varepsilon_{\max }$, una vez que se ha producido la transferencia del pretensado $\left(\varepsilon_{\max , 0}=-545 \mu \mathrm{m} / \mathrm{m}\right)$, se incrementan con rapidez, especialmente durante la primera semana, debido a los fenómenos dependientes del tiempo. Este incremento se va ralentizando y comienza a estabilizarse a partir de los 28-60 primeros días. A los 90 días, las deformaciones totales habían prácticamente triplicado $\left(\Delta \varepsilon_{\max , 90}=173 \%\right)$ la deformación instantánea tras el destesado. Este incremento es coherente con lo obtenido por otros autores $(22,23)$. Aunque el crecimiento de la deformación 
se va atenuando (Figura 8), en vista de los resultados obtenidos se puede afirmar que la influencia de los efectos reológicos no se había detenido en el momento en el que se dejaron de registrar datos.

Las deformaciones de compresión derivadas de los efectos reológicos no sólo elevan el perfil de deformaciones de las vigas, sino que también influyen en la longitud de transferencia $l_{t}$. $\mathrm{Al}$ igual que ocurría con las deformaciones por compresión en la meseta central, las longitudes de transferencia aumentan de forma apreciable los primeros días tras el destesado y la tasa de crecimiento de atenúa con el paso del tiempo, hasta llegar a estabilizarse a partir de los 60 días. A los 7 días la longitud de transferencia se ha incrementado un 7,52\% con respecto a la longitud inicial $l_{t, o}$ derivada de las deformaciones instantáneas, y partir de los 60 días tras el destesado se registran incrementos estables superiores al $15 \%\left(\Delta l_{t, 60}=16,82 \%\right.$; $\left.\Delta l_{t, 9 o}=16,65 \%\right)$.

Por último, la pendiente de los tramos ascendente y descendente del perfil de deformaciones aumenta rápidamente los primeros días tras el destesado y, al igual que ocurre con las deformaciones y las longitudes de transferencia, comienza a estabilizarse a partir de los 28-6o días.

La deformación instantánea ( $\mathrm{t}=\mathrm{o}$ días) de la Figura 8 servirá en el Apartado 5 como base para la comprobación del grado de precisión del modelo analítico.

\subsection{Longitud de transferencia en alambres corroídos}

Tratando de minimizar los efectos reológicos sobre el efecto de la corrosión, el comienzo del proceso de corrosión acelerada se inició un año después de la transferencia del pretensado. La variación de deformaciones que se obtiene en este apartado es con respecto a la viga ya deformada por la transferencia del pretensado y por los efectos dependientes del tiempo.

Para todas las vigas, se han tomado medidas de las deformaciones de compresión en el hormigón durante 42 días, de manera continuada mediante las galgas extensométricas y a intervalos regulares detiempo mediantelos puntos de extensometría manual. Con la densidad de corriente de corrosión $\mathrm{i}_{\text {corr }}$ utilizada $\left(100 \mu \mathrm{A} / \mathrm{cm}^{2}\right)$, este tiempo equivale a pérdidas teóricas de sección de hasta un $10 \%$.

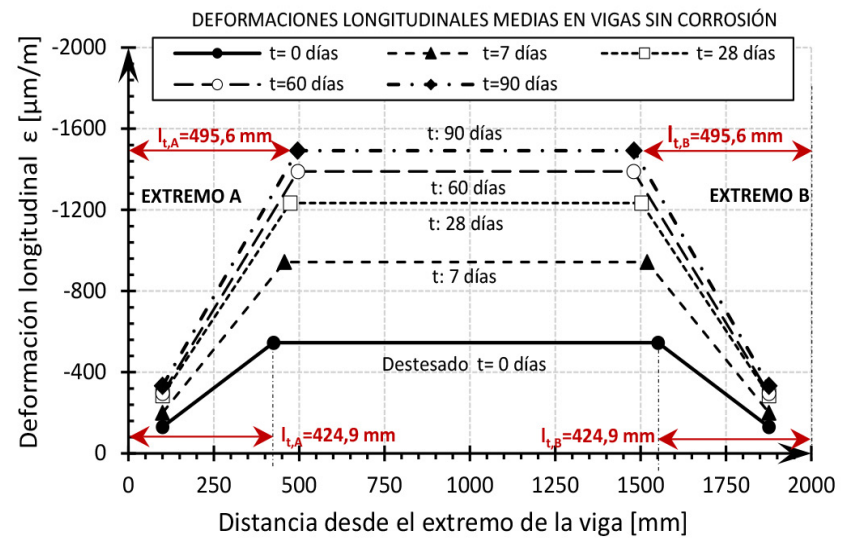

Figura 8. Evolución promedio de las deformaciones longitudinales en todas las vigas ensayadas, tras el destesado. Alambres sin corrosión.
Durante el desarrollo de la campaña experimental, se ha podido comprobar que las medidas tomadas con extensometría manual adolecen de falta de precisión en el caso de deformaciones pequeñas. Este hecho es especialmente significativo en los incrementos de deformación del hormigón derivados del proceso de corrosión, al obtenerse valores bajos en los que los márgenes de error superan las propias medidas obtenidas. Por ello, en este caso, los resultados obtenidos mediante extensometría manual se han obviado en el análisis.

En la Figura 9 se presentan gráficamente los resultados medios de evolución de las deformaciones del hormigón $\varepsilon_{c}$ obtenidos de las galgas extensométricas de las cuatro vigas ensayadas, pudiéndose apreciar con claridad las principales tendencias observadas en los ensayos. Se observa que el mayor deterioro de las propiedades adherentes y, por tanto, la mayor reducción de las deformaciones por compresión del hormigón $\varepsilon_{c}$, se concentra en los extremos de las vigas. Además, dicho deterioro se va atenuando con el aumento de la distancia desde dichos extremos. Este comportamiento es el esperable, dado que las secciones extremas son las más solicitadas por las tensiones radiales $\sigma_{r}$ y circunferenciales $\sigma_{\theta}$, al solaparse el efecto de la corrosión, homogéneo a lo largo de toda la viga, con el efecto Hoyer y el derivado del deslizamiento de las indentaciones, ambos de mayor entidad en los extremos. En estas zonas, la corrosión genera un aumento de dichas tensiones de manera que, al superarse un determinado umbral, el frente de fisuración alcanza la cara externa del recubrimiento, dando comienzo al deterioro de las tensiones radiales $\sigma_{r} \mathrm{y}$, consecuentemente, a la degradación de la tensión de adherencia $\tau_{b}$.

En cuanto a la evolución de las deformaciones del hormigón $\varepsilon_{c}$ en el centro de la viga, se aprecia que para valores bajos de corrosión (1,0-2,5\%) éstas permanecen prácticamente inalteradas. Superados esos niveles de corrosión se observa un incremento de la deformación de compresión $\varepsilon_{c}$ en el centro de vano. Este aumento de las deformaciones no puede deberse en ningún caso al incremento de la fuerza de pretensado, ya que esta fue transmitida previamente, por lo que es achacable únicamente a la acción de los fenómenos reológicos. Sin ser taxativos en este aspecto, ya que no se han monitorizado estos fenómenos, y dado que el efecto de la retracción tendía a estabilizarse tras los tres primeros meses, parece que la fluencia tiene un papel determinante en esta observación.

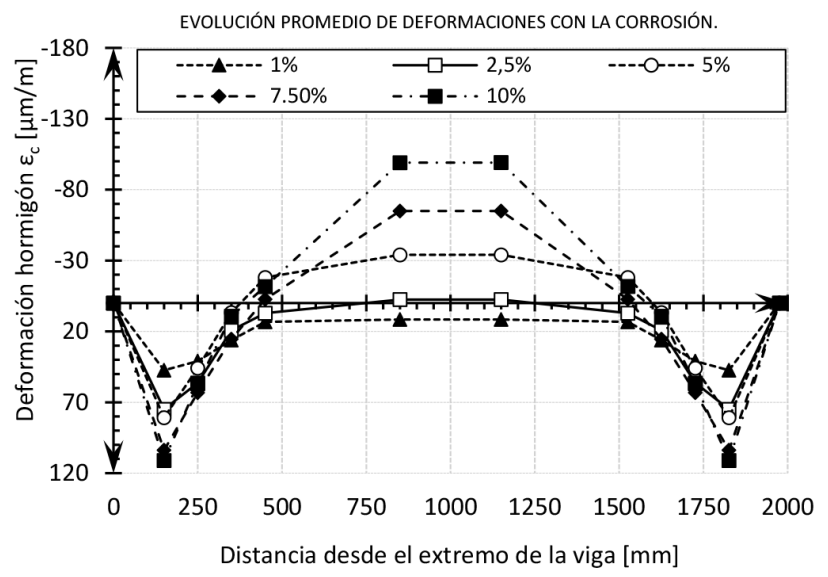

Figura 9. Evolución promedio de las deformaciones longitudinales debidas al proceso de corrosión (pérdidas de sección en \%). 


\section{COMPARACIÓN DE LOS RESULTADOS EXPERIMENTALES CON EL MODELO ANALÍTICO}

En este apartado se realiza la comparación de los resultados obtenidos mediante el modelo analítico descrito en el Apartado 2 con los resultados experimentales registrados en las vigas pretensadas expuestos en el Apartado 4. El análisis de la bondad del modelo se basa en su capacidad de predicción del perfil de deformaciones instantáneas en el hormigón $\varepsilon_{c}$.

El modelo se ha alimentado con los datos geométricos y mecánicos correspondientes a los ensayos sobre vigas pretensadas, expuestos en el Apartado 3, y con el coeficiente de fricción y la ley de tensión de adherencia-deslizamiento calibrados mediante los ensayos de pull-out. En cuanto a las limitaciones del modelo, no se han cuantificado las pérdidas de pretensado ni se han tenido en cuenta los efectos reológicos en el cálculo del perfil de deformaciones, por lo que el modelo es únicamente capaz de determinar las deformaciones instantáneas tras el destesado ( $\mathrm{t}=\mathrm{o}$ días$)$.

\subsection{Longitud de transferencia en alambres sanos}

En vista de estas consideraciones, en la Figura 10 se compara el perfil de deformaciones obtenido mediante el modelo analítico con el ajuste obtenido mediante el Metodo AMS (21) así como con los resultados puntuales obtenidos, tanto mediante medios electrónicos (galgas extensométricas) como manuales (extensometría manual). También se indican las longitudes de transferencia $l_{t}$ obtenidas mediante el análisis AMS de los ensayos y mediante el modelo analítico. En este último caso, y para ser coherente con el análisis de los resultados experimentales, se ha considerado que la longitud de transferencia $l_{t}$ es equivalente a la distancia desde el extremo de la viga hasta el punto en el que se alcanza el $95 \%$ de la deformación máxima de compresión en la meseta central de ésta.

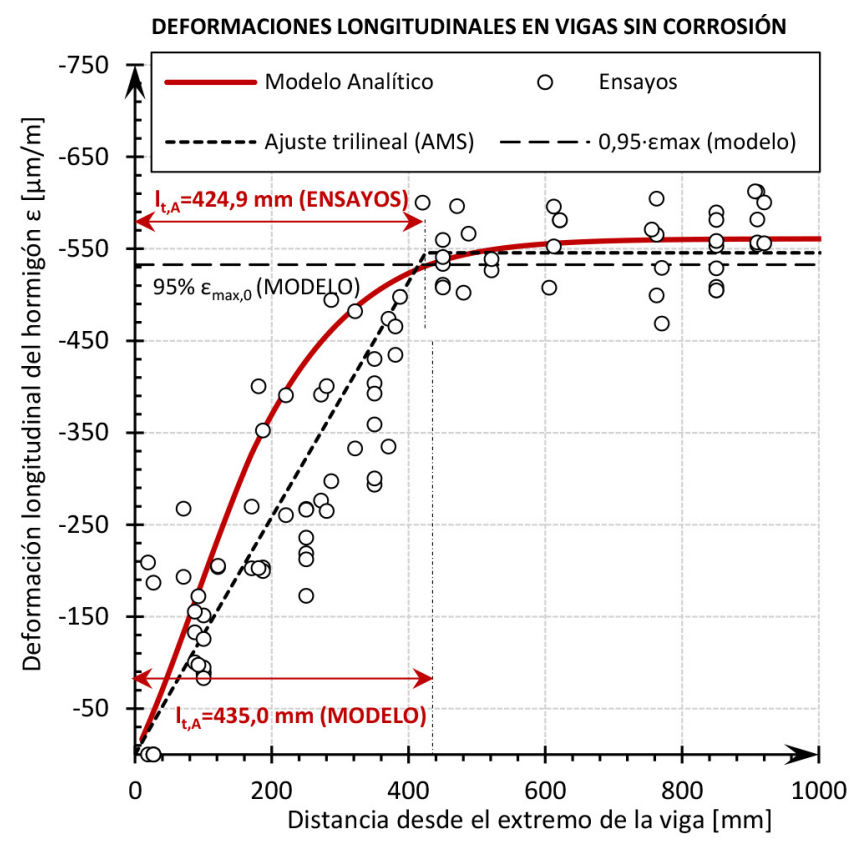

Figura 10. Comparación del perfil de deformaciones instantáneas tras el destesado ( $\mathrm{t}=\mathrm{O}$ días) en los ensayos con los resultados arrojados por el modelo analítico. Alambres sin corrosión.
Tal y como se aprecia en la Figura 10, la deformación máxima instantánea en la meseta central $\varepsilon_{\max }$, que se obtiene de los resultados experimentales ajustados con el Método AMS es estimada correctamente por el modelo analítico. Los resultados experimentales indican que la deformación media instantánea ( $\mathrm{t}=\mathrm{o}$ días) en el tramo central de las vigas, donde el pretensado ha sido transferido completamente al hormigón circundante, es de $-570 \mu \mathrm{m} / \mathrm{m}$, mientras que el modelo predice una deformación de $-561 \mu \mathrm{m} / \mathrm{m}$, lo que supone que el valor predictivo es un 1,7\% menor que el valor real obtenido de los ensayos.

En cuanto a la longitud de transferencia $l_{t}$, el modelo analítico predice que es necesaria una longitud de 435,0 $\mathrm{mm}$ para que la fuerza de pretensado se transmita completamente al hormigón, mientras que los resultados experimentales arrojan un valor 424,9 mm. Esto supone que, para el caso estudiado, el modelo establece un valor de longitud de transferencia un $2,4 \%$ superior al obtenido mediante el análisis de los ensayos.

Las mayores diferencias entre el modelo analítico y los ensayos se aprecian en la pendiente de los tramos ascendente y descendente del perfil de deformaciones. La pendiente de estos tramos es menor en los ensayos, lo que implica que el modelo sobreestima la capacidad de generar tensiones radiales $\sigma_{r}$. Como consecuencia, el modelo predice en estas zonas mayores tensiones de adherencia $\tau_{b}$, que provocan una mayor tasa de transferencia del pretensado al hormigón circundante y, por tanto, una mayor tasa de crecimiento de las deformaciones por compresión en el hormigón. Esta diferencia no es sorprendente, ya que en el análisis de los resultados experimentales se ha asumido, de forma simplificadora, un desarrollo lineal de las deformaciones tanto en el hormigón como en el acero y, por tanto, un valor constante de la tensión de adherencia a lo largo de toda la longitud de transferencia. El efecto Hoyer provoca, desde el extremo y a lo largo de la longitud de transferencia, un gradiente descendente de los desplazamientos radiales de la pared interna del cilindro de hormigón que rodea el alambre y, por tanto, una variación de las tensiones radiales $\sigma_{r} \mathrm{y}$ de la tensión de adherencia $\tau_{b}$.

A la vista de la Figura 10, puede interpretarse que el modelo analítico marca un límite superior del valor de las deformaciones longitudinales en la zona de transferencia, por lo que sobreestima ligeramente la capacidad de confinamiento del recubrimiento y, por tanto, predice una menor fisuración derivada de las tensiones circunferenciales $\sigma_{\theta}$, generadas por la acción conjunta del efecto Hoyer y del deslizamiento de las indentaciones.

A este respecto, es interesante observar cómo evoluciona teóricamente la tensión tangencial $\tau_{b}$ tanto en el ajuste de los resultados experimentales (AMS) como en el modelo analítico. En la Figura 11 se representan ambas tensiones, junto con el avance teórico del frente de fisuración que arroja el modelo. Como puede apreciarse, según el ajuste utilizado, la tensión tangencial $\tau_{b}$ a lo largo de la longitud de transferencia $\left(l_{t}=424,9 \mathrm{~mm}\right)$ toma un valor constante igual a 3,97 MPa. Superada dicha longitud, la tensión de adherencia pasa a ser nula. Por el contrario, según el modelo analítico, la tensión de adherencia $\tau_{b}$ a lo largo de la longitud de transferencia $l_{t}$ pasa por varias etapas. En los primeros $70 \mathrm{~mm}$ desde el extremo, el recubrimiento está completamente fisurado, debido a las tensiones circunferenciales $\sigma_{\theta}$ generadas por la acción conjunta del efecto del pretensado y de las indentaciones en su deslizamiento, pero debi- 


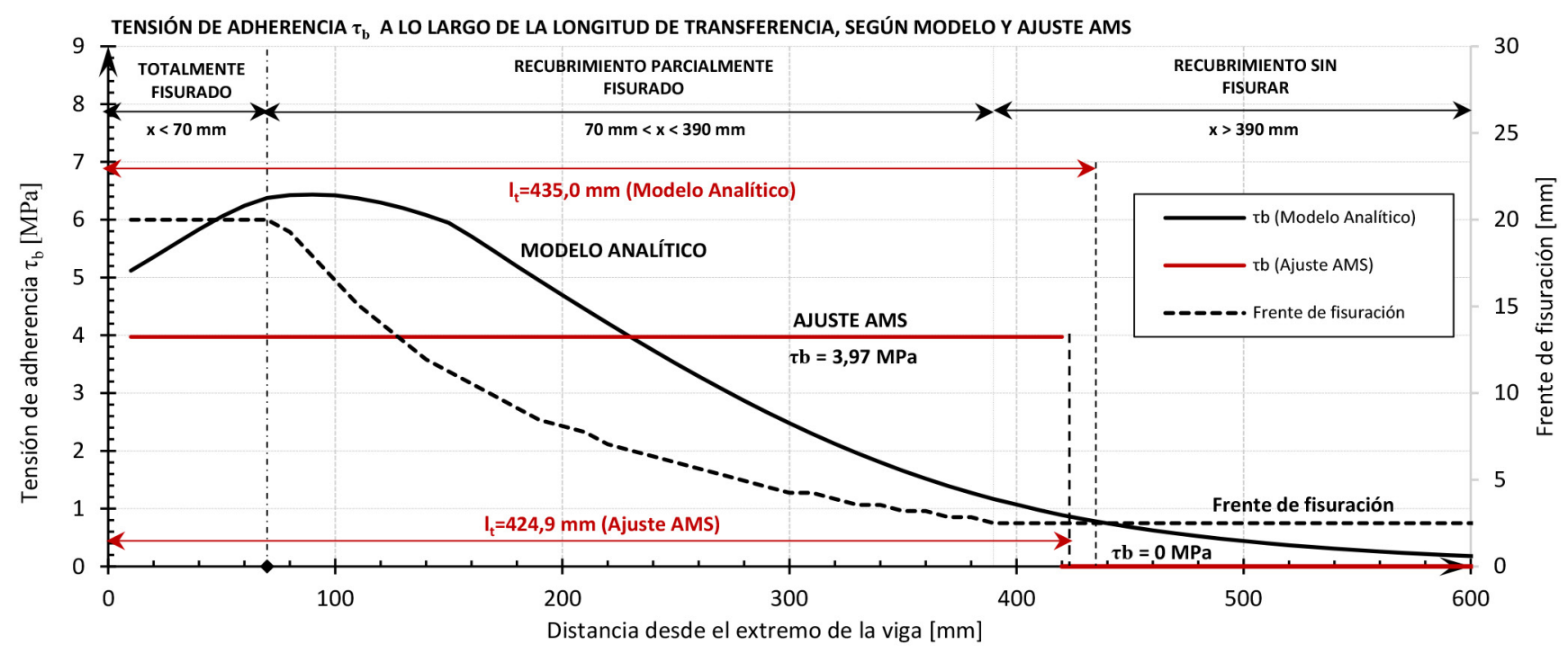

Figura 11. Comparación de las leyes de tensión de adherencia a lo largo de la viga según el modelo analítico y el ajuste AMS de los resultados experimentales. Alambres sin corrosión.

do al modelo de fisuración cohesiva que se ha utilizado, el hormigón es aún capaz de generar tensiones radiales $\sigma_{r}$ considerables. En la segunda etapa, desde el final de la primera hasta una distancia de $390 \mathrm{~mm}$ desde el extremo de la viga, el recubrimiento se encuentra parcialmente fisurado. El frente de fisuración se retrasa, debido a la disminución del desplazamiento radial generado por el efecto Hoyer y al menor deslizamiento de las indentaciones. En esta etapa se alcanza la mayor tensión de adherencia $\left(\tau_{\mathrm{b}, \max }=6,43 \mathrm{MPa}\right)$ a una distancia aproximada de $90 \mathrm{~mm}$ desde el extremo, y comienza a descender hasta alcanzar un valor de $1,17 \mathrm{MPa}$ a una distancia de 390 mm desde el extremo, donde comienza la tercera etapa. En esta etapa el recubrimiento no ha comenzado a fisurarse, por lo que se encuentra en régimen elástico. La tensión de adherencia continúa descendiendo de forma muy atenuada, hasta alcanzar la longitud de transferencia en la que se ha desarrollado el $95 \%$ de las deformaciones $\left(l_{t}=435,0 \mathrm{~mm}\right)$. A partir de ese punto, la tensión de adherencia $\tau_{b}$ residual, generada por pequeños desplazamientos radiales debidos al efecto Hoyer y a las indentaciones, permite alcanzar la transmisión plena de la fuerza de pretensado.

Lógicamente, la integral de la ley de la tensión de adherencia a lo largo de la superficie del alambre en sus respectivas longitudes de transferencia debe ser igual en ambos casos, pero su diferente distribución justifica las diferencias obtenidas en el perfil de deformaciones del hormigón.

\subsection{Longitud de transferencia en alambres corroídos}

A continuación, se lleva a cabo la comparación de los resultados teóricos obtenidos mediante el modelo analítico con los resultados experimentales registrados en las vigas pretensadas con procesos de corrosión. Para los cálculos teóricos se han introducido los valores calibrados en las Fases I y II del modelo (apartados 2.2. y 2.3). Al igual que ocurría con las vigas sin corrosión, no se han cuantificado las pérdidas de pretensado ni se han tenido en cuenta los efectos reológicos en el cálculo de la evolución de las deformaciones, por lo que el modelo analiza de forma aislada la influencia que tiene el proceso de corrosión.
En vista de estas consideraciones, en la Figura 12 se representa la evolución de los incrementos de las deformaciones en el hormigón $\varepsilon_{c}$, obtenidos mediante los ensayos y el modelo analítico. Como puede comprobarse, las diferencias existentes entre los resultados experimentales y el modelo son considerables, de manera que para niveles bajos de corrosión (hasta pérdidas de sección del 2,5\%), el modelo arroja incrementos de deformación significativamente más bajos que los obtenidos en los ensayos $(16,2 \mu \mathrm{m} / \mathrm{m}$ frente a $47,4 \mu \mathrm{m} / \mathrm{m}$, para un $1 \%$ de pérdida de sección), mientras que para niveles medios y altos de pérdida de sección, el modelo predice una relajación en las deformaciones de compresión del hormigón que llega a doblar el resultado experimental $(222,4 \mu \mathrm{m} / \mathrm{m}$ frente a $111,1 \mu \mathrm{m} / \mathrm{m}$ para un $10 \%$ de pérdida de sección teórica).

Por otra parte, los resultados experimentales detectan un incremento de las deformaciones por compresión en el hormi-

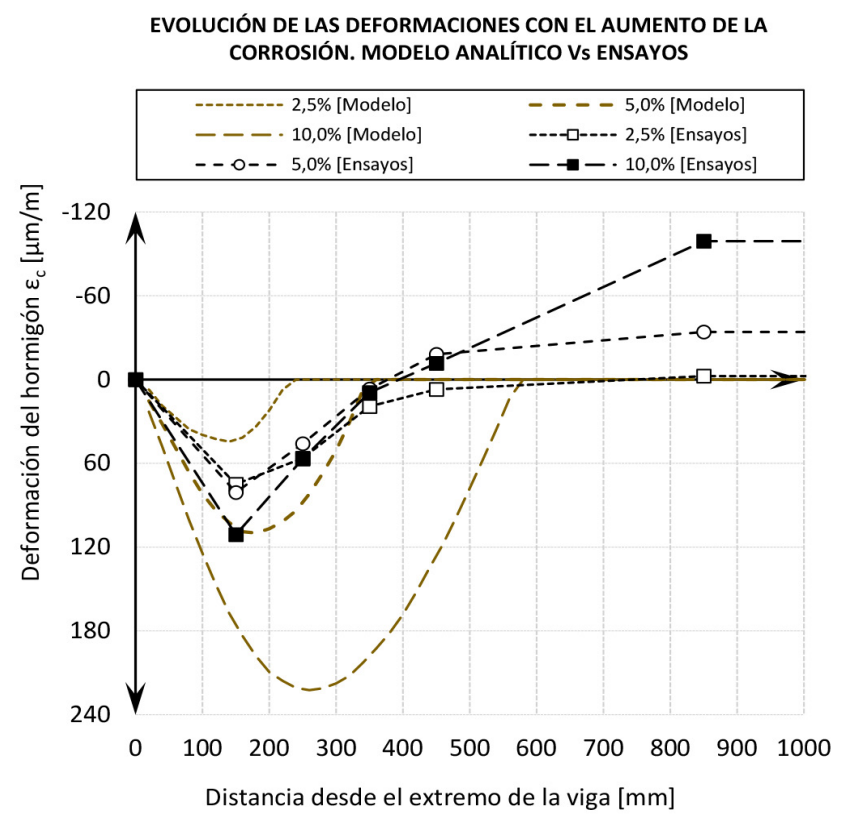

Figura 12. Comparación de la evolución de las deformaciones en el hormigón obtenidas en los ensayos y en el modelo analítico. Alambres con corrosión (pérdidas de sección en \%). 
gón en la zona central de la viga, que aumenta hasta alcanzar un valor de -99,1 $\mu \mathrm{m} / \mathrm{m}$ para una pérdida de sección teórica del $10 \%$. Como se comentó anteriormente, este aumento de las deformaciones de compresión solo puede deberse a los efectos reológicos, y en ningún caso a un aumento de la fuerza de pretensado. Dado que el modelo no contempla los procesos reológicos, era esperable que no se reprodujera este comportamiento.

Partiendo de esta última consideración, si en los ensayos se lograse aislar el efecto atribuible a la corrosión en la variación de las deformaciones por compresión del hormigón, teóricamente se obtendrían resultados más cercanos a los obtenidos en el modelo. Para ello, si se da por cierto que todas las deformaciones que aparecen en el centro de la viga tienen su origen exclusivamente en los fenómenos reológicos, y suponiendo de manera simplificadora que éstos se reparten homogéneamente a lo largo de la longitud de la viga, obtendremos que para cada periodo de tiempo considerado, la variación de las deformaciones de compresión atribuibles únicamente al fenómeno de la corrosión es igual a la diferencia entre el resultado obtenido en los ensayos y el valor de deformación registrado en el centro del vano. En las gráficas de variación de deformaciones en el hormigón, esto sería equivalente a desplazar el eje de abscisas hasta hacer que la deformación en el tramo central de la viga fuera nula. Este ejercicio teórico tiene utilidad porque nos aporta información sobre lo que podría considerarse un límite superior de la variación de deformaciones atribuible a la corrosión, pero en ningún caso pretende ser un reflejo fiel de la realidad, ya que se están suponiendo como ciertas, hipótesis alejadas del comportamiento real. Por ejemplo, mientras que es razonable suponer que la retracción provoca una deformación homogénea a lo largo de toda la viga, no lo es hacerlo con la fluencia, ya que ésta depende del estado tensional de cada sección.

Con todas las salvedades expuestas, en la Figura 13 se comparan las gráficas de evolución de los incrementos de deformación del hormigón, obtenidas del análisis de los ensayos y modificadas para mostrar las deformaciones atribuibles exclusivamente a la corrosión, con los resultados arrojados por el modelo. En la figura se aprecia que el modelo tiene una

EVOLUCIÓN DE LAS DEFORMACIONES CON EL AUMENTO DE LA CORROSIÓN. MODELO ANALÍTICO Vs ENSAYOS

\begin{tabular}{|ll|}
\hline$---\square---2,5 \%[$ Ensayos] & $--0--5,0 \%[$ Ensayos] \\
$-*-\cdot 10,0 \%[$ Ensayos] & $-----2,5 \%[$ Modelo] \\
$----5,0 \%[$ Modelo] & $---\cdot 10,0 \%[$ Modelo $]$ \\
\hline
\end{tabular}

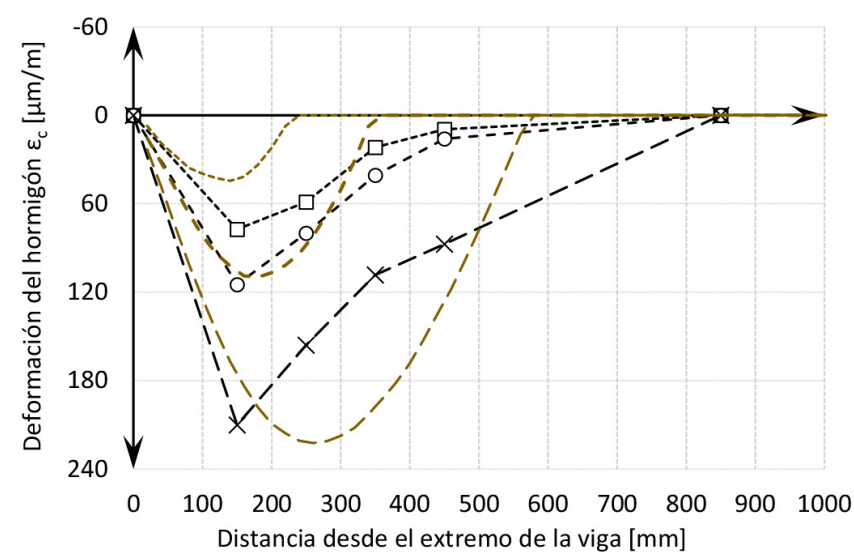

Figura 13. Comparación de la evolución de las deformaciones del hormigón obtenidas de los ensayos (modificadas para aislar el efecto de la corrosión) y del modelo analítico. Alambres con corrosión (pérdidas de sección en \%). buena capacidad predictiva. Para valores medios y altos de pérdida de sección (5-10\%) los máximos arrojados por el modelo son muy similares a los obtenidos en los ensayos, mientras que se observa una desviación mayor para valores bajos de corrosión (1-2.5\% de pérdida de sección). Para éstos últimos, el modelo es muy sensible a pequeñas variaciones en parámetros como el espesor equivalente de la capa porosa $t_{p, c r i}$ o el módulo de compresibilidad volumétrica $K_{r}$, por lo que no es sorprendente que las mayores diferencias se localicen en etapas tempranas del proceso de corrosión.

En definitiva, teniendo en cuenta la heterogeneidad del proceso de corrosión, por un lado, y el volumen de asunciones y de simplificaciones en las que se basa el modelo analítico, por el otro, puede afirmarse que el modelo propuesto es capaz de detectar las principales tendencias observadas en los ensayos (deterioro concentrado en los extremos, reducción del deterioro hacia el centro de la viga, etc) y arroja resultados que captan el orden de magnitud del fenómeno.

\section{CONCLUSIONES}

En este trabajo se ha desarrollado un modelo analítico integral para la determinación de la longitud de transferencia en estructuras pretensadas con armaduras pretesas sanas y corroídas. El modelo ha sido verificado mediante una campaña experimental, diseñada siguiendo la Norma UNE 7-436-82 (12).

La campaña experimental sobre vigas con alambres sanos ha permitido extraer las siguientes conclusiones:

- Las longitudes de transferencia aumentan de forma notoria los primeros días tras el destesado, atenuándose con el paso del tiempo hasta llegar a estabilizarse a partir de los 60 días, con un incremento medio de aproximadamente un $16 \%$

- Tras la deformación instantánea de la viga, tiene lugar un incremento rápido de las deformaciones por compresión, especialmente significativo la primera semana, debido a los fenómenos de retracción y fluencia. Este incremento se va ralentizando y comienza a estabilizarse a partir de los 28-6o primeros días. A los 90 días, las deformaciones totales aproximadamente se han triplicado, debido a la contribución de los efectos reológicos. Consecuentemente, la pendiente de los tramos ascendente y descendente del perfil de deformaciones aumenta rápidamente los primeros días tras el destesado y, al igual que ocurre con las deformaciones y las longitudes de transferencia, comienza a estabilizarse a partir de los 28-6o días.

Del proceso de corrosión acelerada de las vigas se derivan las siguientes observaciones:

- El mayor deterioro de las propiedades adherentes y, por tanto, la mayor reducción o relajación de las deformaciones por compresión del hormigón, se concentra en los extremos de las vigas, ya que son las regiones más solicitadas antes del comienzo del proceso de corrosión, desde el punto de vista de tensiones radiales $\sigma_{r}$ y circunferenciales $\sigma_{\theta}$, debidas al pretensado y al deslizamiento de las indentaciones.

- Las deformaciones del hormigón $\varepsilon_{c}$ en el centro de la viga permanecen inalteradas para valores bajos de pérdida de sección (1,0-2,5\%). Superados esos niveles de corrosión, 
se produce un apreciable aumento de la deformación de compresión en el centro del vano. Este incremento de las deformaciones de compresión no puede deberse en ningún caso al incremento de la fuerza de pretensado, ya que esta fue transmitida previamente, ni al efecto de la corrosión, por lo que es achacable únicamente a la acción de los fenómenos reológicos de retracción y fluencia.

Finalmente, de la verificación del modelo analítico, mediante su comparación con los resultados experimentales, se extraen las siguientes conclusiones:

- El modelo analítico desarrollado, basado en la teoría de cilindros de pared gruesa bajo presión interna uniforme y con fisuración cohesiva del hormigón, presenta una capacidad predictiva de las deformaciones instantáneas en vigas pretensadas con alambres sanos que puede calificarse como muy buena.

- En el caso de procesos de deterioro por corrosión, el modelo es capaz de detectar las principales tendencias observadas en los ensayos, como el deterioro concentrado en los extremos y la reducción del éste hacia el centro de la viga, arrojando resultados que captan el orden de magnitud del fenómeno.

En definitiva, en este trabajo se ha verificado que el modelo analítico desarrollado es una herramienta útil para el análisis de vigas pretensadas con alambres sanos y corroídos. En artículos posteriores se mostrará como esta herramienta ha permitido el estudio cuantitativo de la influencia de las distintas variables que intervienen en el fenómeno, así como el desarrollo de expresiones simplificadas para el cálculo de longitudes de transferencia y su evolución con el avance de los procesos de deterioro por corrosión, contribuyendo a cubrir el vacío normativo existente en la actualidad.

\section{AGRADECIMIENTOS}

Los autores agradecen al personal técnico de la Nave de Ensayos del IETcc todas las aportaciones realizadas y las facilidades ofrecidas durante la realización de los ensayos, dada la complejidad de la campaña experimental acometida. Igualmente, agradecen a R. Soldado y a J. A. Morales su colaboración en la toma de medidas de las deformaciones de las vigas pretensadas.

El Dr. Pablo Anaya Gil desea agradecer la financiación recibida para la realización de este trabajo de investigación, en forma de becas predoctorales, por parte del Consejo Superior de Investigaciones Científicas (CSIC), mediante su programa JAE-Predoc, y de la Comisión Europea, mediante el proyecto NOVA DOMUS, perteneciente al programa ERASMUS MUNDUS.

\section{REFERENCIAS}

(1) Andrade, C., Alonso, C., Molina, F. (1993). Cover cracking as a function of bar corrosion: Part I-Experimental test. $M a-$ terials and Structures, 26(8): 453-464. https://doi.org/10.1007/BFo2472805

(2) Rodríguez, J., Ortega, L. M., García, A. M. (1994). Assessment of structural elements with corroded reinforcement. En Congreso Internacional Corrosion and Corrosion Protection of Steel in Concrete (pp. 171-185). Sheffield, Reino Unido: Sheffield Academic Press.

(3) ACI Comittee 318 (2002). Building Code Requirements for Structural Concrete (ACI 318-2) and Commentary (318Ro2). American Concrete Institute.

(4) Comisión Permanente del Hormigón (2008). Instrucción Española de Hormigón Estructural (EHE-o8). Madrid: Ministerio de Fomento.

(5) FIB (2012). Model Code for Concrete Structures 2010. Fib Bulletin 65-66. Fédération Internationale du Béton (fib)

(6) CEN (2004). EN 1992-1-1:2004. Eurocode 2: Design of concrete structures. General rules and rules for buildings. Comité Europeo de Normalización (CEN).

(7) Mitchell, D., Cook, W., Khan, A. (1993). Influence of High Strength Concrete on Transfer and Development Length of Pretensioning Strand. PCI Journal, 38(3): 52-66.

(8) Marti-Vargas, J.R., Arbelaez, C.A., Serna-Ros, P., Navarro-Gregori, J., Pallares-Rubio, L. (2007). Analytical model for transfer length prediction of $13 \mathrm{~mm}$ prestressing strand. Structural Engineering and Mechanics, 26(2): 211-229. https:// doi.org/10.12989/sem.2007.26.2.211

(9) Oh, B.H., Lim, S.N., Lee, M.K., Yoo, S.W. (2014). Analysis and Prediction of Transfer Length in Pretensioned, Prestressed Concrete Members. ACI Structural Journal, 111(3): 549-56o. http://doi.org/10.14359/51686571

(10) Anaya Gil, P. (2020). Influencia de la corrosión en la adherencia de alambres en elementos pretensados de hormigón (Tesis doctoral). Universidad Politécnica de Madrid (UPM).

(11) AENOR-AEN/CTN 36 (2006). UNE-EN 10o8o:2006 “Acero para el armado del hormigón. Acero soldable para armaduras de hormigón armado. Generalidades. Asociación Española de Normalización (AENOR).

(12) IRANOR (1982). UNE 7-436-82. Método de ensayo para la determinación de las características de adherencia de las armaduras de pretensado. Instituto Nacional de Racionalización y Normalización (IRANOR).

(13) Timoshenko, S. (1956). Strength of Materials. Part II: Advanced Theory and Problems. Princeton, New Jersey: D.Van Nostrand Company Inc.

(14) Tepfers, R. (1973). A theory of bond applied to overlapped tensile reinforcement splices for deformed bars. Goteborg, Suecia: Chalmers University of Technology.

(15) Bažant, Z.P. (1979). Physical model for steel corrosion in concrete sea structures-theory. ASCE Journal of the Structural Division, 105(6): 1137-1153.

(16) Pantazopoulou, S.J., Papoulia, K.D. (2001). Modeling Cover-Cracking due to Reinforcement Corrosion in RC Strucrtures. Journal of Engineering Mechanics, 127(4): 342-351. https://doi.org/10.1061/(ASCE)0733-9399(2001)127:4(342). 
(17) Oh, B.H., Kim, E.S., Choi, Y.C. (2006). Theorical Analysis of Transfer Lengths in Pretensioned Prestressed Concrete Members. Journal Engineering Mechanics, 132(10): 1057-1066. http://doi.org/10.1061/(ASCE)O7339399(2006)132:10(1057)

(18) Molina, F., Alonso, C., Andrade, C., (1993). Cover cracking as a function of rebar corrosion: Part 2-Numerical model. Materials and Structures, 26(9): 532-548. https://doi.org/10.1007/BFo2472864

(19) Andrade, C. (2013). Especificaciones de cálculo de la vida útil y estado límite de corrosion. Revista ALCONPAT, 2(3): 79-97. https://doi.org/10.21041/ra.v3i2.45

(20) AENOR (2012). UNE 83992-2:2012 EX Durabilidad del hormigón. Métodos de ensayo. Ensayos de penetración de cloruros en el hormigón. Parte 2: Método integral acelerado. Asociación Española de Normalización (AENOR).

(21) Russell, B. \& Burns, N., (1997). Measurement of transfer lengths on pretensioned concrete elements. Journal of Structural Engineering, 123(5): 541-549. https://doi.org/10.1061/(ASCE)0733-9445(1997)123:5(541)

(22) Oh, B.H., Kim, E.S. \& Kim, K.S. (2001). Correct Prediction of Transfer Lengths in Pretensioned Prestressed Concrete Structures. Trabajo presentado en SMiRT 16 Conference. Washington DC, Estados Unidos.

(23) Vázquez-Herrero, C., Martínez-Lage, I., Martínez-Abella, F. (2013). Transfer length in pretensioned prestressed concrete structures composed of high performance lightweight and normal-weight concrete. Engineering Structures, 56: 983992. https://doi.org/10.1016/j.engstruct.2013.06.020 